\title{
A Value-Focused Approach for Establishing Requirements Specification of Commercial Aircraft
}

\author{
Xinwei Zhang ${ }^{1}$, Shurong Tong ${ }^{1}$, Hakki Eres ${ }^{2}$, Mario Kossmann ${ }^{3}$, Keqin Wang ${ }^{1}$ \\ ${ }^{1}$ Institute of Design Management, School of Management, Northwestern Polytechnical University, \\ Xi' an, 710072, China; ${ }^{2}$ Faculty of Engineering and the Environment, University of Southampton, \\ Highfield, Southampton, SO17 1BJ, UK; ${ }^{3}$ Airbus, Bristol, UK.
}

\begin{abstract}
Although Systems Engineering (SE) processes and standards are widely used in aircraft development programs, traditional requirements engineering practice for commercial aircraft does not explicitly address value perceptions and associated information. In this paper, a value-focused approach is proposed to promote a better understanding of customer value perceptions and their derivation among different levels for value-based requirements engineering of commercial aircraft. The approach is a four-step process starting from initial customer statements to a customer value model, and leading to a system value model with associated component value models. A set of theories and methods are introduced in order to resolve different aspects of the approach regarding the appropriate understanding of customer value perceptions and the establishment of the value-based requirements specification. A case study is used to demonstrate the transformation of airlines' initial expectation statements into three types of value models. There are two significant benefits of this approach: 1) perceived customer value can be explicitly modeled, simulated and derived into different levels of the system development; and 2) the value model can subsequently be utilized reactively for design evaluations and proactively for design optimization to generate creative design alternatives.
\end{abstract}

Keywords: requirements, value model, value-driven design, commercial aircraft, requirements engineering 


\section{Introduction}

During the initial phrases of a new commercial aircraft program, many conceptual decisions are made. These primarily include early product positioning, subsequent definition of top-level aircraft objectives (or technical requirements at aircraft level), and distributing these objectives within the extended enterprise hierarchy (aircraft, engine, and subsystems manufacturers). Ideally, all of these decisions are based on their assumed value contribution to airline customers and/or other relevant internal or external stakeholders. However, this assumed value contribution is more an implicit concept, rather than an explicit consideration in aircraft development programs. Furthermore, value related information such as value dimensions, their measurement and value trade-offs that allow for early conceptual orientation at the different development levels within the extended enterprise are not usually explicitly shared between the partner organizations.

Systems Engineering (SE) processes and standards are widely used in the aircraft development programs, however they do not address "value" in much detail at the Requirements Engineering (RE) stage $^{1-3}$. Airlines' expectations and needs are usually collected and converted into technical requirements at the aircraft level in a value-implicit manner. That is, how these aircraft technical requirements influence airlines' value is not quantified sufficiently. These aircraft level technical requirements are then flowed down to component level requirements, such as engine level requirements, using approaches of decomposition and derivation. A complex set of processes, methods and tools are available in literature and applied in the aerospace industry to support and fulfill the task of requirements establishment. This set mainly includes, quality function deployment (QFD), use cases, customer focus groups, viewpoint analysis and functional analysis methodology ${ }^{4-6}$. However, this paradigm of flowing down aircraft technical requirements into lower levels in an aircraft hierarchy is error-prone and it can be inconsistent with airlines' own value perceptions. Examples of this kind of value conflict have been identified by Collopy ${ }^{7}$. Also, they have been identified as one major source of large value loss, leading to significant corrective rework, and related additional costs and delays.

Examples of ignoring a holistic and value-focused viewpoint are also widespread in the aircraft design and evaluation process ${ }^{8}$. For instance, aircraft design alternatives are traditionally evaluated in terms of recurring and non-recurring costs by aircraft manufacturers and direct/indirect operating costs by airlines or other operators. More recently, surplus value (or profit) based system value models have 
been proposed to optimize aero-engine and fuselage designs in the value-driven design community, which enables surplus value produced from commercial aircraft operations as the single objective function or decision criterion ${ }^{9,10}$. Evidently, cost or profit is one special and critical dimension of value, but there are other dimensions, such as performance, safety, reliability, cabin comfort and many others. All these dimensions are potentially perceived as valuable by customers and other stakeholders. If too much attention is paid to one single dimension or some special dimensions, it is difficult to make beneficial value trade-offs between different dimensions. This will further fail to provide high-value aircraft to airline customers and other stakeholders. Researchers from MIT had developed multi-attribute trade-space exploration paradigm for conceptual design of aerospace systems, delivering value to key stakeholders ${ }^{11,12}$. The concept of 'value' in this trade-space exploration paradigm is explicit through plotting utility compared with total lifecycle cost in a two-dimensional space; while utility is preference-based on a decision-maker's perceived metrics or attributes. Their intention is for trade-space exploration in a system level for a given set of design alternatives, without communicating value-related information into lower-level system development. But, obviously, it would be better to use value-related information at different levels of system requirements and design.

In this paper, a value-focused approach is proposed that helps to better understand customer value perceptions for value-based RE. It is a four-step process from understanding customer expectations to derive component value models with supporting methods, such as multi-attribute utility theory, means-ends analysis and part-whole analysis. The approach is applied in order to understand airlines' value perceptions for the development of value-based requirements specifications of commercial aircraft. This contributes to the following three aspects: (1) Value can be explicitly qualified (what it is and what are its internal structural relationships), quantified (how much or its measurement), modeled (in terms of value models), and simulated (e.g. using the Monte-Carlo simulation) in the design and development of commercial aircraft; (2) airline value models and their derived lower-level value models can be used to evaluate available or potential commercial aircraft concepts, and thereby enhance the decision-making process of selecting aircraft design alternatives; and (3) airline value models and their derived lower-level value models can be communicated among different development levels within the extended enterprise, with an emphasis on highly influencing value dimensions and value drivers for designing alternatives with high value perceptions.

This paper is organized as follows. In Section 2, the context in which the proposed approach was 
developed is introduced. In Section 3, the value-focused approach to support commercial aircraft RE is proposed with its process, and underlying theories and methods. Section 4 provides insights on the application of the four-step approach to a simplified example to engineer a value-based requirements specification for commercial aircraft, transforming airlines' initial statements of expectations into three kinds of value models. Finally, Section 5 summarizes the conclusions and future research directions are identified.

\section{Background}

\subsection{Value and Value model}

In the paper, the $3^{\text {rd }}$ definition of value listed in Merriam-Webster Online dictionary is used ${ }^{13}$ :

Relative worth, utility, or importance $<$ a good value at the price $><$ the value of base stealing in baseball $><$ had nothing of value to say $>$.

This definition is much broader than those value definitions that only focus on profit, cost, or any other monetary value. It also conforms to the definition of value in decision analysis context in which value always has multiple dimensions. In a commercial aircraft context, the acquisition cost of commercial aircrafts is enormous and the number of customers is relatively small compared to general consumer products. Aircraft manufacturers must consider particular value perceptions of individual customers or individual categories of customers; therefore they usually have a direct dialogue with their customers in order to better capture customer value ${ }^{14}$. These characteristics determine that the approaches of understanding customer value for commercial aircraft are different from those approaches for general consumer products. Besides customer value, value perceptions from an extended enterprise of the aircraft manufacturer, e.g. from system designers, component suppliers and other stakeholders, are also essential to requirements establishment, but they are not considered here for the sake of simplicity.

A value model is a quantitative objective function developed from measurable attributes of interest through verifying independence assumptions among them ${ }^{15}$. These attributes of interest and their achievement are of value to decision makers in a particular context. Customer value model, system value model and component value models are established from customer attributes, system attributes, and component attributes, respectively. These value models, such as the customer value 
model, are multi-dimensional in nature, and can reflect all aspects that are perceived as valuable in the aircraft development context. Therefore, they are broader than traditional, economic value models, e.g. profit or surplus-based value models ${ }^{9,16}$.

\subsection{Context of Approach Development}

Commercial aircraft manufacturers face ever-increasing pressures to develop operationally economical, comfortable and high-performance commercial aircraft to satisfy the needs and expectations of customers and other stakeholders. These pressures compel or lead the manufacturers to develop more capability on Modeling and Simulation to improve the virtual development process of commercial aircraft. One work package of requirements establishment and value generation within the European Commission's seventh framework (FP7) research project CRESCENDO in the domain of European aerospace industry was dedicated to develop approaches of value-based requirements engineering, which enables value-based requirements modeling and simulation. This work package provided a beneficial link to main manufacturers (i.e., aircraft, engine, sub-systems and others) in the European aerospace industry and useful input during the development of the approach. The developed approaches and value models in this work package, when integrated with other engineering models in the commercial aircraft development cycle, contributed to value-driven design of commercial aircraft.

A schematic illustration of value-driven design (VDD) among different levels of aerospace product is presented in Figure $1^{9,17}$. In this framework, a system value model is a multi-attribute utility function rather than the surplus value model deployed in the original investigation ${ }^{9}$. The system value model is rather a combination of customer (airlines are the only customers and fare paying passengers are not considered here) value model and interface model that models the functional relationships between customer needs and top-level aircraft requirements. With the system value model it is straightforward to calculate the utility of special inputs of aircraft attributes that are outputs of aircraft product model. When the system value model is implemented, evaluation of different design alternatives and optimization studies in terms of customer value becomes a possibility. This capability is attributed to single attribute utility information and value trade-off information contained in the system value model. This VDD cycle in Figure 1 is from sub-component models through component models, product model, and system value model back to sub-component models. It is actually very flexible, and models can be added to extend sub-component models to further finer models, such as the sub-sub-component models. Low-level engineering models in Figure 1 can be moved from 
sub-component models into component models. For example, deletion of the part of "sub-component model" will create an evaluation and optimization cycle from component models to product model, to system value model, and finally back to component models.

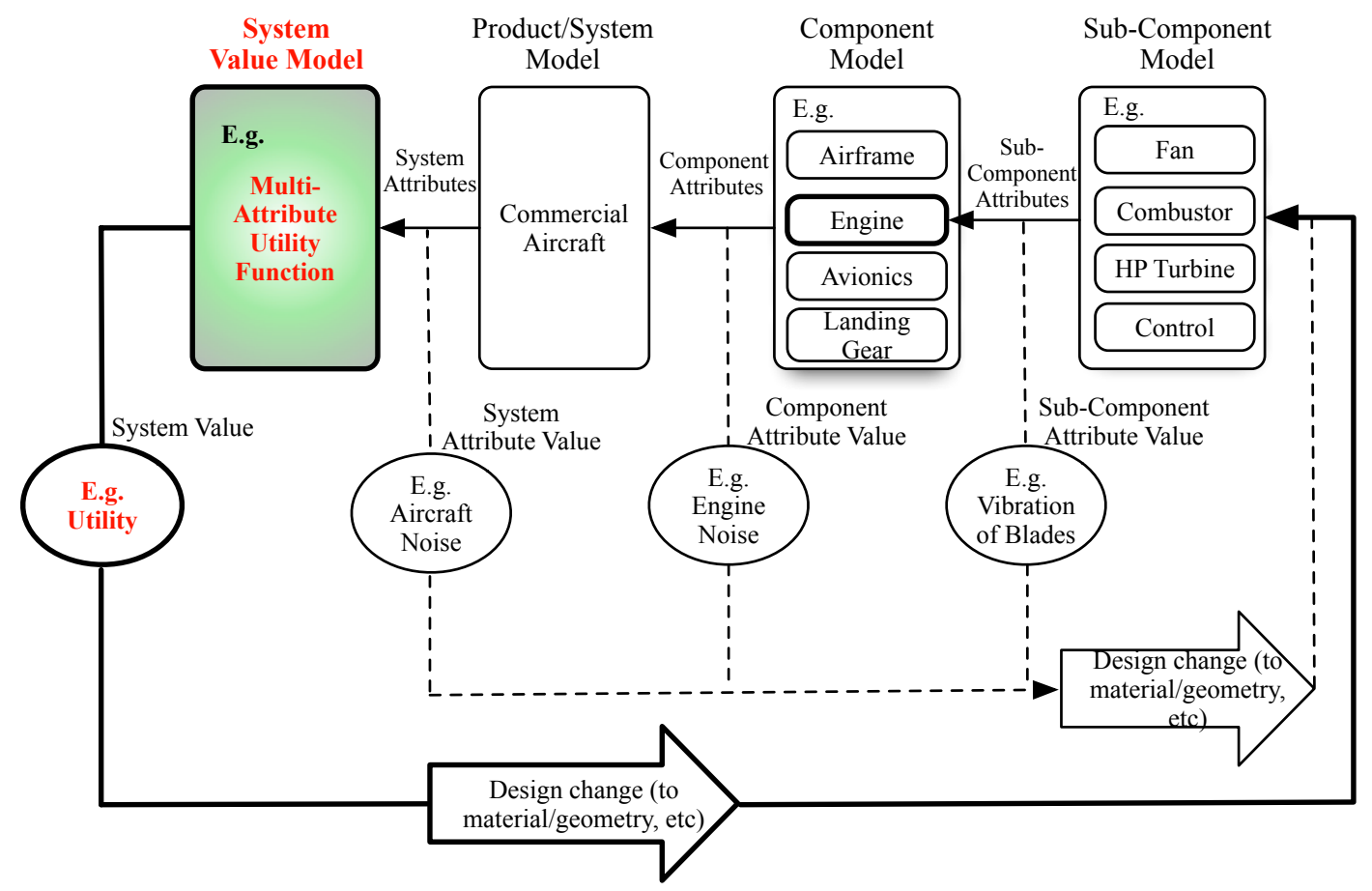

Figure 1. A schematic illustration of VDD in context of CRESCENDO 9,17

One innovative aspect of the work package is how to build three types of value models from initial customer statements of needs, expectations, goals, and constraints in parallel with the traditional RE process, which ensures that the developed aircraft reflects customer value. It includes:

- How to build the airline value model,

- How to build the aircraft value model,

- How to build the associated component value models (and their sub-component value models).

When these value models are appropriately constructed, they can be added to the traditional requirements specification, specifically to their corresponding levels of requirements in the aircraft development process as presented in Figure 2. 


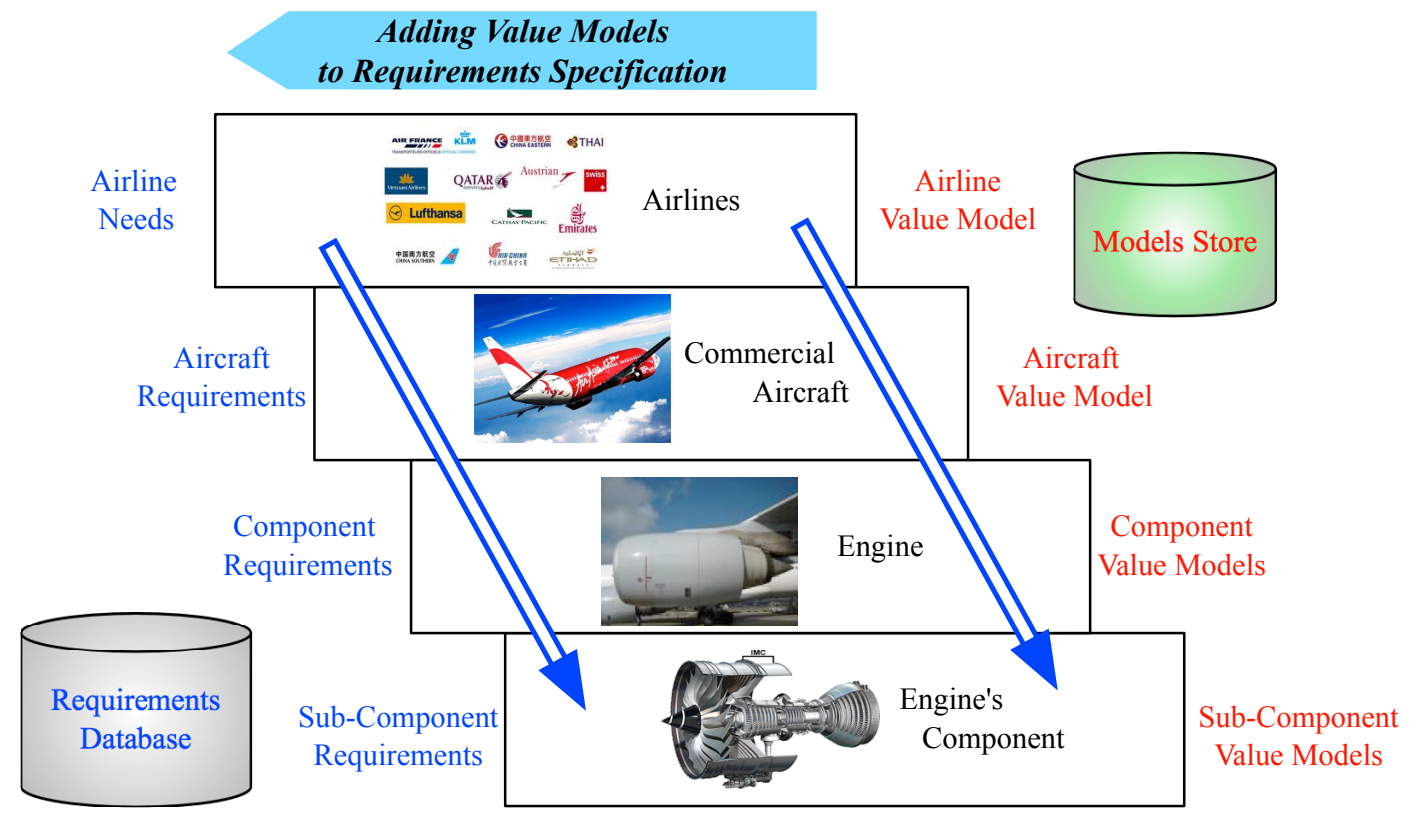

Figure 2. Adding value models to traditional requirements specification

Furthermore, there are several dozen layers in the commercial aircraft development process. Thus, value models should be built for every level of the requirements establishment. Indeed, the logic and solution of constructing sub-component value models from component value model is the same as that of constructing component value models from system value model. The same situation is also intuitive and obvious for lower levels of sub-components. Therefore, in order to demonstrate the approach, it is reasonable to focus only on the first three layers of value models.

\section{The Approach}

A value-focused approach is proposed to transform initial customer statements into three types of value models. In order to transform initial customer statements into a customer value model, customer needs that are perceived as fundamentally important in the current commercial aircraft development context need to be identified as thoroughly as possible. Additionally, these customer needs have to be structured to some extent in order to enable identifying and selecting measurable customer attributes. The customer value model is then built from this set of attributes with multiple attributes-based value methods. While customer attributes for measuring customer needs are solution-independent, customer attributes and system attributes that are solution-dependent and technical need to be mapped. This mapping and its functional relationships are the premise of developing a system value model consistently with the customer value model. The system value model is a function of valuation using 
system attributes as parameters, while the system model (say, product model in Figure 1) is available to model the function how component (e.g. aero-engine) attributes contribute to system attributes. Therefore, it is straightforward to obtain a function that represents how a component attribute influences the system value. Finally, the system value model is decomposed into a set of component value models that are objective functions built from component attributes.

The proposed approach includes the following four steps as shown in Figure 3, which are described using the IDEF0 modeling language. IDEF0 uses boxes to represent activities and lines with arrows to link the activities where the arrows indicate the direction of flow.

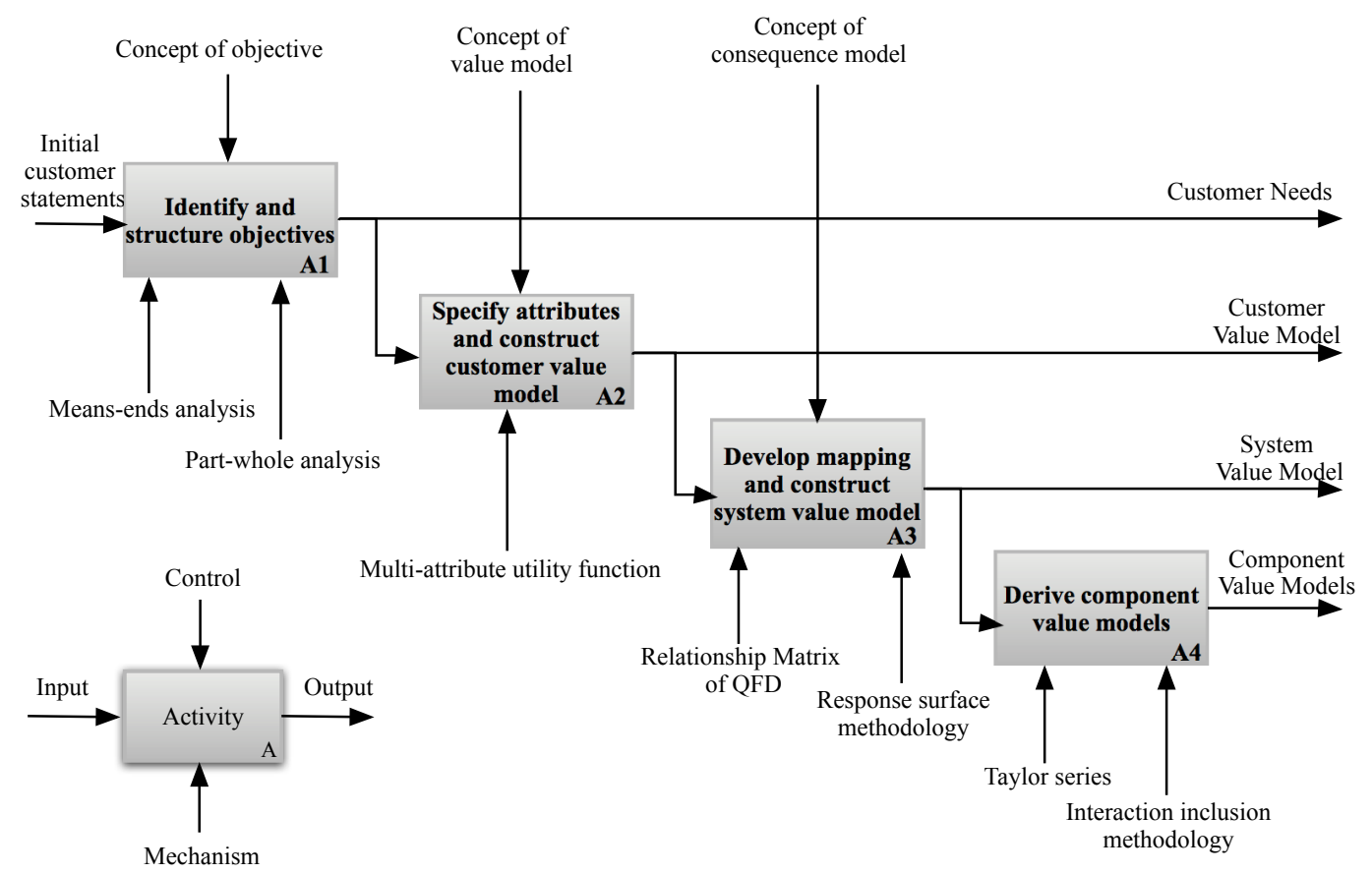

Figure 3. The four steps of the value-focused approach

A1. Identify and structure objectives to find real customer needs.

The main aim of this step is to identify what is of value to customers in the current context of system development. The value may be elicited as needs, goals, constraints, system requirements, proposed technology or design alternatives with different formats of expression, levels in system hierarchy or granularities of information. These various types of customer statements may hinder a deeper understanding of customer values to some extent. In order to reduce potential problems, these initial customer statements are then transformed into a common grammatical expression of 
verb plus noun form. For example, 'minimize' is the verb and 'cost' is the noun in the objective of 'minimize cost'. They are then called objectives and classified into three categories of means objectives, fundamental objectives (customer needs) and strategic objectives with means-ends relationships ${ }^{18}$. Customer needs are fundamentally important and solution-independent in the current context. They are classified as fundamental objectives. System requirements, proposed technology, or design alternatives are means objectives to influence the achievement of customer needs. Whether goals or constraints are means, fundamental or strategic objectives need to be analyzed and decided on a case-by-case basis.

Means-ends analysis is performed to create corresponding, logical structures of a means-ends objectives network and pursue the customer needs in the current context. Through means-ends analysis, customer needs that are implicit in the means objectives are discovered, and customer needs in the context are identified as thoroughly as possible. With these identified customer needs, part-whole analysis is deployed to clarify the meaning of customer needs and to further improve the understanding. This part-whole analysis helps to obtain a hierarchy of customer needs. This hierarchy explains customer needs with a finer granularity, and it will later facilitate the process of identifying measureable attributes of customer needs.

A2. Specify customer attributes for customer needs and construct customer value model.

Attributes are specified to measure the attainment levels of customer needs. With measurable attributes, it is then possible to transform qualitative value perceptions into a quantitative value model. As measurable attributes are often missed during traditional RE practices, processes and methods are needed to carefully identify and select appropriate attributes. This is by no means a trivial task, but it is absolutely necessary. Various traps should be avoided in order to identify a set of attributes satisfying the set of desired properties of attributes as defined by Keeney ${ }^{19}$.

A customer value model is then constructed based on the verified independence assumptions among the set of attributes, which is a multi-attribute utility function. The underlying theory to support the quantification and to construct a customer value model is the 'multi-attribute utility theory ${ }^{20}$. Given a set of $N$ identified customer attributes $X=\left(X_{1}, X_{2}, \ldots, X_{i} \ldots, X_{N}\right)$, if mutual utility independence can be verified among $X$, 


$$
1+w u(x)=\prod_{i=1}^{n}\left[1+w w_{i} u_{i}\left(x_{i}\right)\right]
$$

is an appropriate measurement of value with regards to a special achievement $x=\left(x_{1}, x_{2}, \ldots, x_{i} \ldots, x_{N}\right)$ of $X$, where $u_{i}$ is a single attribute utility function over attribute $X_{i}$ scaled from 0 to 1 and $w$ 's are scaling constants. More variants of equation (1) are available according to verified independent assumptions among $X$. Specially, if $\sum_{i=1}^{n} w_{i}=1$, a linear additive function form is found.

A3. Develop mapping and construct system value model.

Customer needs and their attributes should be mapped into system attributes that are of technical nature and that will influence the attainment of customer needs. This step is necessary to understand how the system requirements contribute to the customer value perception. While this step seems similar to the transformation step in the house of quality, mathematical rigor is needed to model high-order effects of system attributes and effects of interactions between system attributes on customer attributes when these effects are apparent. These effects cannot be modeled with the linear additive function form in the relationship matrix. The underlying method to establish a functional form between customer attributes and system attributes is response surface methodology $\mathrm{y}^{21}$. Given the system has a set of $M$ system attributes $Y=\left(Y_{1}, Y_{2}, \ldots, Y_{J} \ldots Y_{M}\right)$, a mapping function from system attributes $Y$ to customer attribute $X_{i}$ is found; hence

$$
\mathrm{x}_{\mathrm{i}} \approx \beta_{\mathrm{i} 0}+\sum_{\mathrm{j}=1}^{\mathrm{M}} \beta_{\mathrm{ij}} \mathrm{y}_{\mathrm{ij}}^{\prime}+\sum_{\mathrm{j}=1}^{\mathrm{M}} \beta_{\mathrm{ijj}}\left(\mathrm{y}_{\mathrm{ij}}^{\prime}\right)^{2}+\sum_{\mathrm{j}=1}^{\mathrm{M}} \sum_{\mathrm{l}>\mathrm{j}}^{\mathrm{M}} \beta_{\mathrm{ijl}} \mathrm{y}_{\mathrm{ij}}^{\prime} \mathrm{y}_{\mathrm{il}}^{\prime}
$$

Where $\beta_{i j}$ are regression coefficients for the first degree terms, $\beta_{i j j}$ are coefficients for the quadratic terms, and $\beta_{i j l}$ are coefficients for the cross-product terms, and $\beta_{i 0}$ is the intercept term.

Alternatively, the mapping and the underlying functions can sometimes be found directly in engineering practice, such as the surplus value model and reliability model in a commercial aircraft context. This established function form (2) or existing engineering models, when combined together with a customer value model, such as the equation (1), helps establishing a 
system value model $u_{y}$.

A4. Derive component value models from system value model.

In this step component value models are derived from system value model through performing a sensitivity analysis. These component value models are used for component design, optimization and evaluation similar to requirements. This step is different from the traditional requirements engineering practices of requirements flowing down from system level to components level. The transition from requirements flow-down to value model flow-down is to avoid possible methodological problems of inconsistent decision-making between the system level and the component level.

Assume a system value model $u_{y}$ being sufficiently smooth in its attribute space. It can be linearized by a Taylor series expansion that is composed of a linear function of all component attributes $^{7}$. A component value model $u_{p}$ of the component $p$, e.g. aero-engine, can be obtained as:

$$
u_{p} \approx \sum_{k=1}^{m_{p}}\left(\sum_{j=1}^{M} \frac{d u_{y}}{d y_{j}}\left|y_{j}^{\prime} \cdot \frac{d y_{j}}{d z_{k}}\right| z_{p k}^{\prime}\right) \cdot z_{k}
$$

Where $m_{p}$ is the number of attributes of the component $p, z_{k}{ }^{\prime} s, k=1, \ldots, m_{p}$, are attributes of the component $p$. Thus, a component value model is then a linear function of its component attributes. This is one convenient derivation of component value model from system value model.

In the next section, an application of the approach is deployed for transforming airlines' initial expectation statements into an airlines' value model, an aircraft value model, and an aero-engine value model through a simplified but illustrative example.

\section{Application}

After a new commercial aircraft program has been initiated and positioned in an aircraft product family, airlines' common expectations are elicited in customer-focus groups from all potential customers while special expectations are elicited in bilateral meetings from customers ${ }^{3}$. These shared common 
expectations are usually about aircraft emission levels, operational reliability, maintenance and operational costs, improved fuel consumption, and so on. The special expectations are usually about the way of operating the cabin and searching for commonalities (e.g. for aero-engines) within a fleet of aircraft from different aircraft manufacturers. Therefore, the elicited common expectations are the core input for identifying common needs and for requirements establishment. In particular, there are similar value perceptions about the common needs for one category of customers, e.g. low-cost airlines or flagship airlines. It is then reasonable to assume that the airlines in the same category have the same value perceptions or that a common value perception can be achieved after discussions.

After customer-focus groups, a large set of common airline expectations has been elicited, concerning different levels in aircraft hierarchy and different granularities of gathered information. These expectations are first transformed into grammatical expressions of "objective" and then a means-ends analysis is performed. Two kinds of questions are frequently asked to pursue the underlying cause-effect relationships, such as "why is fuel consumption important?" and "how can fuel consumption be reduced?" The fundamental reasons of interest in the current context are then finally discovered as completely as possible, which constitutes a hierarchy of high-level airlines' needs as shown in Figure 4. For example, "maximizing profitability" and "fit flight mission" are customer needs because they are fundamentally important and they are final ends in the context. However, "maximize maintainability" and "maximize reliability" are customer needs from one perspective, and are means to influence customer needs from another. They are means because they influence the attainment of profitability while they are customer needs because there are other aspects of them except profit, e.g. physical and mental influence that are important to airlines. Thus, they are included in the hierarchy only to mean those aspects rather than profit, and their influence on profit are already included in "maximizing profitability". This separation is necessary to reduce of the possibility of double counting. 


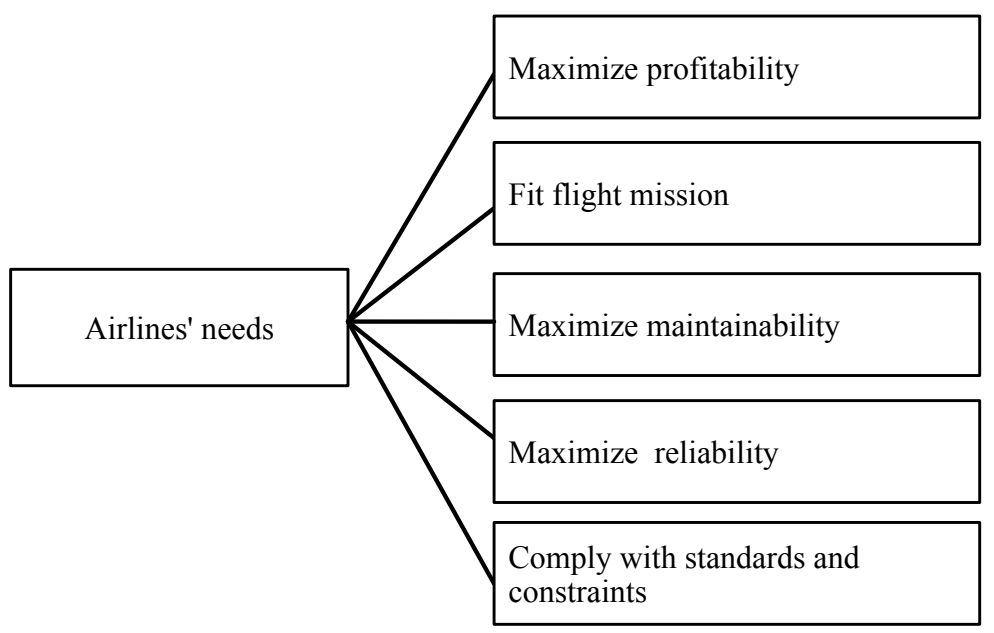

Figure 4. A hierarchy of high-level airlines' needs

The airlines' needs in the hierarchy are at a high level with a coarse granularity. It is necessary to perform part-whole analysis to clarify their meaning. Questions, such as "What do you mean by complying with standards and constraints?" are usually asked. The answer to this question reveals that the standards are about emission and noise and that the constraints are about airworthiness authorities' directives and safety requirements. Further questions can be asked about the answer of the former question in order to have a clear understanding. This clarification of the meaning of airline's needs helps identifying attributes for measuring their attainment. The identified and selected customer attributes for each need are listed in Table 1.

Table 1. Customer attributes for measuring the achievement of customer needs

\begin{tabular}{|l|l|}
\hline Airlines' needs & Attributes for measuring \\
\hline Maximize profitability & $X_{1}$ : Surplus value measured in 2013 millions dollars \\
\hline Maximize maintainability & $X_{2}:$ Mean maintenance man-hours per flight hour \\
\hline Fit flight mission & $X_{3}$ : Yes or No \\
\hline Maximizing reliability & $\begin{array}{l}X_{4}: \text { The probability that the scheduled flight leaves within a specified } \\
\text { delay time }\end{array}$ \\
\hline $\begin{array}{l}\text { Comply with standards and } \\
\text { constraints }\end{array}$ & $X_{5}$ : Yes or No \\
\hline
\end{tabular}

After specifying attributes, it is time to identify possible preference independence assumptions among them, which helps finding functional forms of the value model, e.g., multiplicative or additive functional forms. For example, in order to identify the relationships between surplus value $\left(X_{1}\right)$ and man-hours per flight hour $\left(X_{2}\right)$, a test as in Figure 5 is conducted. For all values of $X_{1}$ and $X_{2}$, the customer is indifferent between these two lotteries, which implies the existence of additive independence between $X_{1}$ and $X_{2}$. When additive independence is identified, an additive function can 
be used to compose $X_{1}$ and $X_{2}$. For $M$ attributes, the same procedures are used, although it can be time-consuming when $M$ is large.

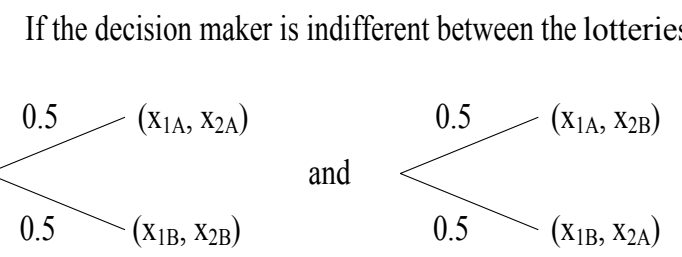

for all values of $x_{1}$ and $x_{2}$, then $x_{1}$ and $x_{2}$ are additive independence.

Figure 5. Verification of independence assumptions

Empirically, Keeney shows that after carefully performing means-ends analysis, part-whole analysis and selecting appropriate attribute for each need ${ }^{18}$, the equation

$$
u\left(x_{1}, \ldots, x_{M}\right)=\sum_{i=1}^{M} k_{i} u_{i}\left(x_{i}\right)
$$

is a reasonable approximation of customer preferences customer preferences.

Single attribute utility functions for attributes are then assessed. Necessary information for assessing one single attribute utility function includes: 1) range information of the attribute, 2) the monotonicity of the utility function, 3) risk attitude of the customer towards the uncertainty attainment of the attribute, and 4) certainty equivalence (or lottery equivalence). One hypothetical single attribute utility function (i.e. surplus value) is modeled and illustrated in Figure 6. For a single attribute utility function for surplus value, the underlying process is the following: (1) Collect range information of the attribute, that is from 10 to 90 million dollars of surplus value, (2) Ascertain the direction of increasing preferences, and identify those that are monotonically increasing, (3) Identify airline risk attitudes towards the uncertain attainment of surplus value and risk averse is found, (4) Select the class of risk averse utility functions ${ }^{18}$ such that

$$
u_{i}\left(x_{i}\right)=a+b\left(-e^{-c x_{i}}\right)
$$


where $a$ and $b>0$ are constants and are selected to ensure $u_{i}$ being scaled from zero to one, constant $c>0$ indicates monotonically increasing preferences, and the value of $c$ indicates the degree of airline's risk averseness, (5) Assess certainty equivalence (or) lottery equivalence, and 3500 million dollars are determined as being indifferent to the lottery $\langle 1000,0.5,8000\rangle$ which yields either a 1000 or a 8000 million dollars surplus value, each with a $50 \%$ chance, and (6) Solve $a$, $b$ and $c$ in equation (5) to determine a single attribute utility function over surplus value using available information.
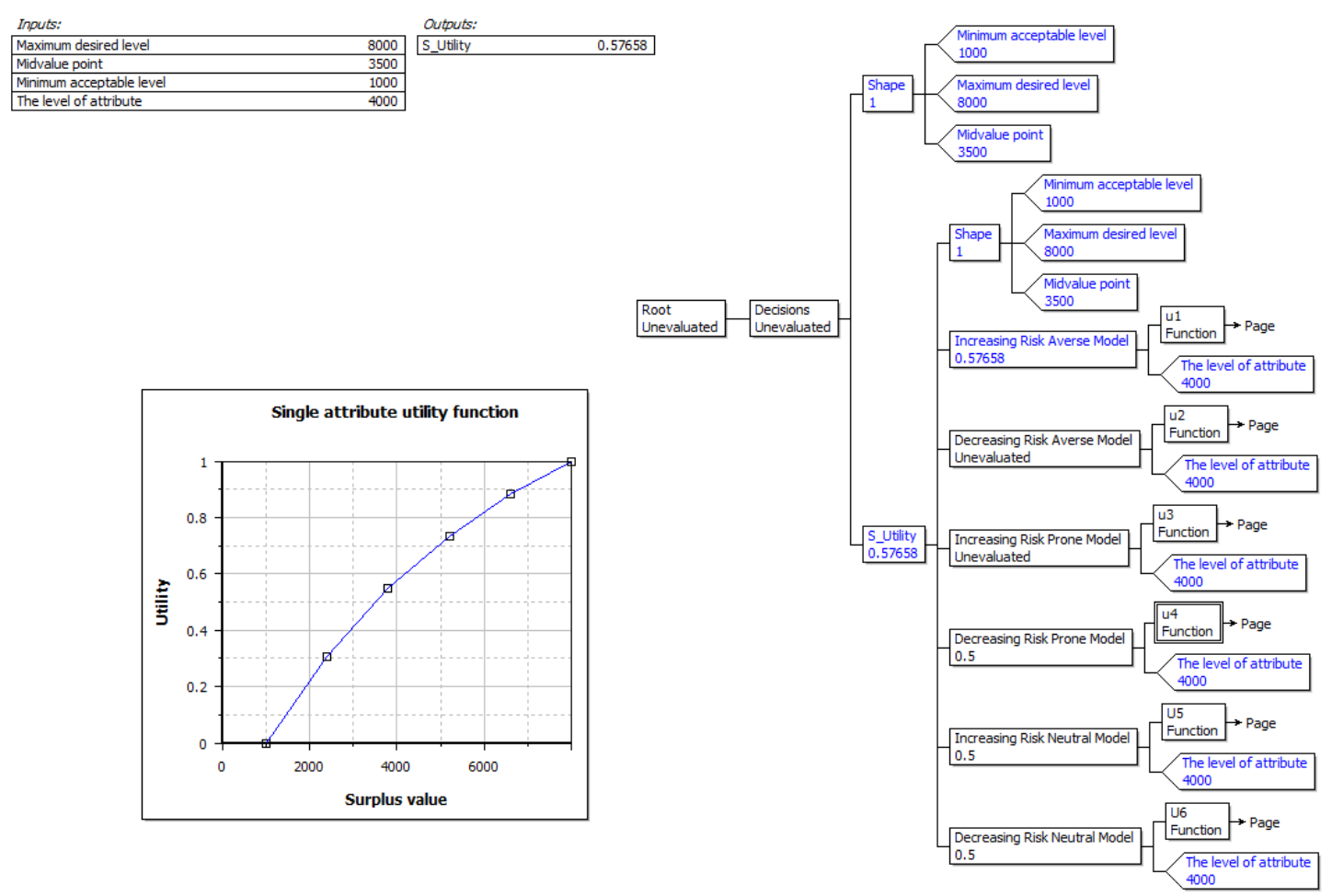

Figure 6. Single attribute utility function over surplus value

These assessments of single attribute utility functions and following value models are implemented in the Vanguard Studio ${ }^{\circledR}$, which is a hierarchical software tool for decision analysis and has a wide range of applications. A Vanguard Studio model has been developed for the assessment of single attribute utility function, which includes six sub-models:

- Increasing risk averseness model,

- Increasing risk neutrality model,

- Increasing risk proneness model,

- Decreasing risk averseness model, 
- Decreasing risk neutrality model,

- $\quad$ Decreasing risk proneness model.

The inputs of the model are following (when monotonicity is verified):

- $\quad$ The most desired level of the attribute $x_{i}^{\prime}$,

- The minimum acceptable level of the attribute $x_{i}^{\prime \prime}$,

- The mid-value point in the attribute range $x_{i}^{\prime \prime \prime}$.

Then the model can judge the direction of increasing preferences, customer's risk attitudes and assess the single attribute utility automatically. The underlying rationale is that when there are the inputs of $x_{i}^{\prime}, x_{i}^{\prime \prime}$ and $x_{i}^{\prime \prime \prime}$, three equations can be found. For example, if $x_{i}^{\prime}>x_{i}^{\prime \prime \prime}$, then the single attribute utility function is increasing; if $x_{i}^{\prime \prime}<\frac{x_{i}^{\prime}+x_{i}^{\prime \prime \prime}}{2}$, then the customer is risk averse over the attribute, which gives the following equations:

$$
\begin{gathered}
u_{i}\left(x_{i}^{\prime}\right)=a+b\left(-e^{-c x_{i}^{\prime}}\right)=1 \\
u_{i}\left(x_{i}^{\prime \prime}\right)=a+b\left(-e^{-c x_{i}^{\prime \prime}}\right)=0.5 \\
u_{i}\left(x_{i}^{\prime \prime \prime}\right)=a+b\left(-e^{-c x_{i}^{\prime \prime \prime}}\right)=0
\end{gathered}
$$

After some manipulation, these three nonlinear equations are transformed into three single-variable equations (9), (10) and (11). And they are solved in Vanguard Studio ${ }^{\circledR}$ to determine the three parameters.

$$
\begin{gathered}
2 e^{-c x_{i}^{\prime \prime}}=e^{-c x_{i}^{\prime}}+e^{-c x_{i}^{\prime \prime \prime}} \\
b=\frac{1}{e^{-c x_{i}^{\prime \prime \prime}}-e^{-c x_{i}^{\prime}}}
\end{gathered}
$$




$$
a=b e^{-c x_{i}^{\prime \prime \prime}}
$$

The constants $k_{i}{ }^{\prime} s$ in the equation (4) are determined by making value trade-offs and constructing equations. For example, if an airline would like to reduce surplus value from 40 million dollars to 30 , in order to reduce man-hours per flight hour from 0.3 to 0.1 , then

$$
u\left(40,0.3, x_{3}, x_{4}, x_{5}\right)=u\left(30,0.1,3, x_{4}, x_{5}\right)
$$

is found. With three mutual independent equations (as $k_{3}=0$ and $k_{5}=0$ ), all unknown constants are solved. With all single attribute utility functions and constants, airlines' value model is established.

The next step is to identify and relate the functions from aircraft requirements to customer needs. In our simplified case, engineering models are already available, e.g. a surplus-value model and a reliability model. The aircraft value model can be directly established by combing the airlines' value model with these engineering models. With the established aircraft value model, component value models are finally derived according to equation (3). In order to derive the engine value model from the aircraft value model, the engineering requirements are connected with aircraft requirements based on experience, response surface modeling or literature research. The engine value model is then obtained by first order partial derivatives of the aircraft value model with respect to the aircraft requirements and the first order partial derivatives of aircraft requirements with respect to aero-engine requirements. The implemented aero-engine value model is shown in Figure 7. It is then possible to quantify how the aero-engine requirements influence the engine value. This is achieved by changing the input of aero-engine requirements in the value model. Also, their different levels of influence are displayed with a different color to better communicate this information to support design decisions. 

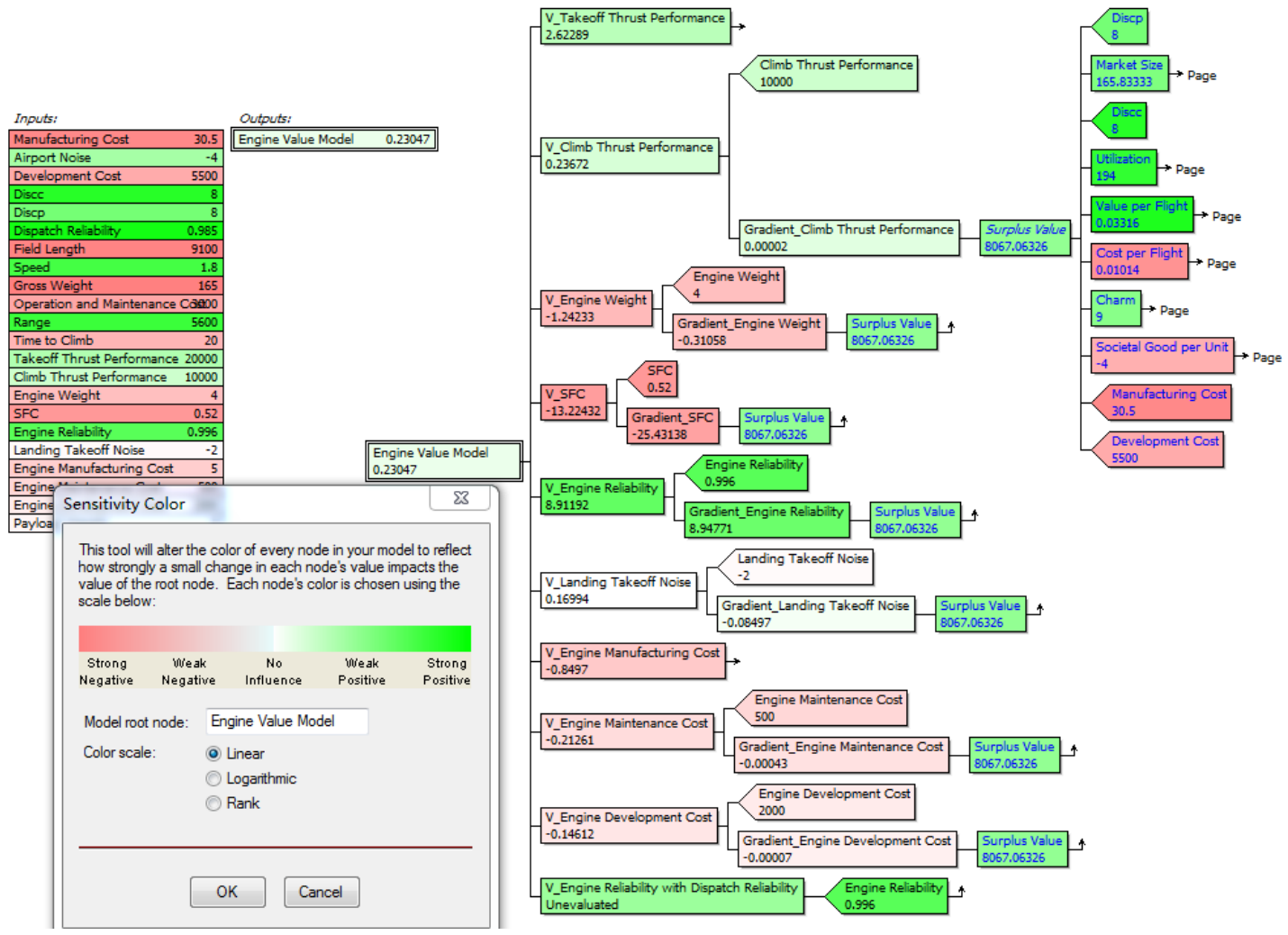

Figure 7. The derived engine value model

While these value models are implemented in Vanguard Studio ${ }^{\circledR}$, many types of capabilities of the tool are utilized, for example, surface plots, optimization, component-based modeling, Internet-based simulation and Monte Carlo simulations. Figure 8 shows a surface plot demonstrating how range and manufacturing cost influence aircraft value. 


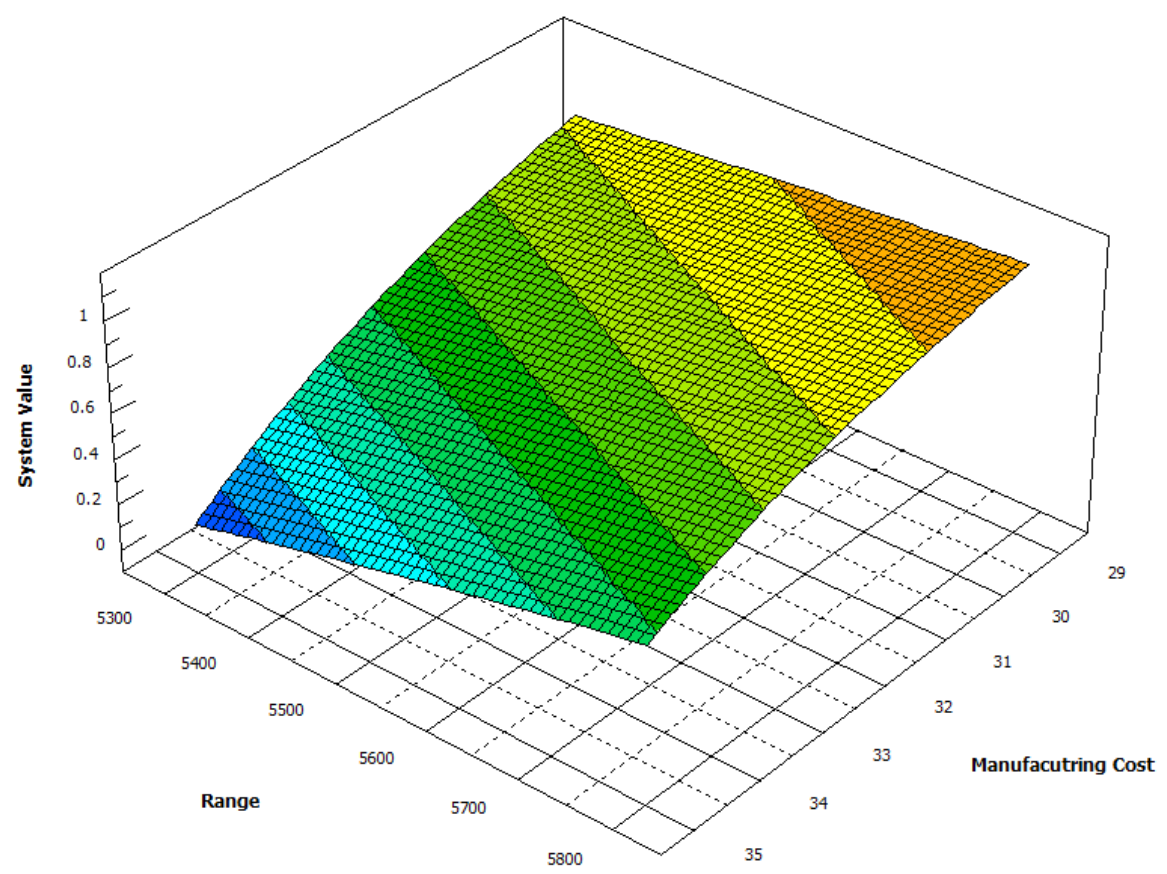

Figure 8. Surface plot of aircraft value against range and manufacturing cost

\section{Conclusions}

In this paper we proposed a value-focused approach to understand customer value perceptions for value-based RE and a case study of transforming airlines' initial expectation statements into three types of value models. The approach is a four-step process integrating the existing 'multi-attribute utility theory', 'means-ends analysis', 'part-whole analysis', 'response surface methodology' and others. These theories and methods were introduced to resolve different concerns appearing during the different steps of the approach. With the approach and the illustrated case study, we identified the following benefits:

- Airlines' multi-dimensional value perceptions can be explicitly modeled and simulated, which was presented through the case study in Section 4. These value perceptions and relevant value models were added to traditional requirements specification enabling value-based RE of commercial aircraft. Therefore, value-based RE and value models can be integrated into existing quantitative engineering models for value-driven design of commercial aircraft.

- Aircraft value model can be used to evaluate available or potential aircraft alternatives, when the necessary information of aircraft concept alternatives is collected as shown in Figure 1. A scalar 
value is calculated for the set of information attributes of a specific aircraft alternative. This is a very attractive feature that enhances the decision-making process.

- As the component value models are derived from aircraft value model, they are consistent with the airline value model. This process enables developing components through distributed design while optimizing airline value perceptions.

- Value models can also be used for the conceptual orientation at all different development levels within the extended enterprise, when the validated technical requirements are not available. They are used for actively designing towards value with the contained information within themselves, such as the value dimensions, their relative weights and single attribute utility functions. This can reduce development risks throughout the extended enterprise, and reduces the 'time to market' of new aircraft.

The future research should focus on a number of aspects regarding simulations across multiple levels of development within the extended enterprise; the configuration management of the related value-based information at all levels concerned; and the re-use of this information, including traceability information, for major modification projects or new aircraft programs. Also, different approaches to derive value models from the system level to the component level should be further investigated to compare their respective performance, applicability and limitations. Comparative studies should be conducted between requirements flow-down and value model flow-down in different development contexts. Finally, temporal and dynamic characteristics of value models should be considered.

\section{Acknowledgements}

The research leading to these results has received funding from the European Community's Seventh Framework Programme (FP7/2007-2013) (www.crescendo-fp7.eu) under grant agreement n॰234344 and from Northwestern Polytechnical University (13GH0311, GDKY2011). The authors would like to thank Guillaume Auriol and Claude Baron of INSA Toulouse, as well as Anne Monceaux from EADS Innovation Works for the many fruitful discussions they had with them during the last years. 


\section{References}

1. ISO/IEC. ISO/IEC 15288:2008(E), Systems and software engineering - System life cycle processes. ISO/IEC-IEEE, 2008.

2. Hull E, Jackson K and Dick J. Requirements Engineering. 3nd ed. London: Springer, 2011.

3. De Chazelle P, Cornes M and Kerrien A. Customer Focused Engineering in Airbus A380 Programme. 14th Annual INCOSE International Symposium. Toulouse, France: INCOSE, 2004.

4. Pahl G and Beitz W. Engineering Design: A Systematic Approach. London: Springer, 2007.

5. Ulrich K and Eppinger S. Product Design and Development. 4th ed.: McGraw-Hill/Irwin Press, 2008.

6. Lempia D and Miller S. Requirements Engineering Management Handbook Federal Aviation Administration, 2009.

7. Collopy PD and Hollingsworth PM. Value-Driven Design. J Aircraft. 2011; 48: 749-59.

8. Jenkinson L, Simpkin P and Rhodes D. Civil Jet Aircraft Design. Arnold \& AIAA, 1999.

9. Cheung J, Scanlan J, Wong J, et al. Application of Value-Driven Design to Commercial Aeroengine Systems. J Aircraft. 2012; 49: 688-702.

10. Castagne S, Curran R and Collopy P. Implementation of value-driven optimisation for the design of aircraft fuselage panels. Int J Prod Econ. 2009; 117: 381-8.

11. Ross AM, Hastings DE, Warmkessel JM and Diller NP. Multi-attribute tradespace exploration as front end for effective space system design. J Spacecraft Rockets. 2004; 41: 20-8.

12. Ross AM, McManus HL, Rhodes DH and Hastings DE. Revisiting the Tradespace Exploration Paradigm: Structuring the Exploration Process. AIAA Space 2010. Anaheim, CA: AIAA, 2010.

13. Merriam-Webster. 2013.http://www.merriam-webster.com/dictionary/value

14. Ross AM, O'Neill MG, Hastings DE and Rhodes DH. Aligning Perspectives and Methods for Value-Driven Design. AIAA Space 2010. Anaheim, CA: AIAA, 2010.

15. Edwards W, F. Miles R and von Winterfeldt D. Advances in Decision Analysis. 1st ed.: Cambridge University Press, 2007.

16. Hazelrigg GA. An axiomatic framework for engineering design. J Mech Design. 1999; 121: $342-7$.

17. Zhang XW, Auriol G, Eres H, Baron C and Kossmann M. Understanding Airlines' Value Perceptions for Value-Based Requirements Engineering Of Commercial Aircraft. 22nd Annual INCOSE International Symposium. Rome: INCOSE, 2012.

18. Keeney RL. Value-Focused Thinking. Cambridge, Massachusetts: Harvard University Press, 1992.

19. Keeney RL and Gregory RS. Selecting attributes to measure the achievement of objectives. Operations Research. 2005; 53: 1-11.

20. Keeney RL and Raiffa H. Decisions with Multiple Objectives: Preferences and Value Tradeoffs. Cambridge Press, 1993.

21. Myers RH, Montgomery DC, Vining GG, Borror CM and Kowalski SM. Response surface methodology: A retrospective and literature survey. J Qual Technol. 2004; 36: 53-77. 